\title{
Superconductivity and Quantum Critical Behavior in $\mathrm{Cr}_{100-z} \mathrm{Os}_{z}$
}

\begin{abstract}
A.R.E. Prinsloo*, C.J. Sheppard And P.R. Fernando
Physics Department, Science Faculty, University of Johannesburg, Auckland Park, Johannesburg, South Africa

The magnetic phase diagrams of Cr alloys with group- 8 metals, such as Re and Ru, show interesting properties. These include quantum critical and superconducting behavior. In order to extend these investigations this paper reports on the physical properties and the magnetic phase diagram of the $\mathrm{Cr}_{100-z} \mathrm{Os}_{z}$ system, investigated in a concentration range of $2 \leq z \leq 30.6$. Structural analysis indicate that samples with $z<22$ have a bcc structure, while those with $z>22$ include a cubic A15-type structure. Transport and magnetic property measurements indicate that the antiferromagnetic phase disappears at $z \approx 14$ and that samples with concentrations $z \geq 12.2$ show superconducting behavior. The magnetic phase diagram of $\mathrm{Cr}_{100-z} \mathrm{Os}_{z}$ is constructed from the Néel and superconducting transition temperatures obtained from various measurements. The commensurate spin-densitywave to paramagnetic phase line decreases sharply for $z>4$. Fitting parameters of a power law fit to the commensurate spin-density-wave to paramagnetic phase line indicate possible quantum critical behavior. Present results suggest that this phase line terminates in a superconducting dome near a magnetic quantum critical point, similar to that observed in certain heavy fermion systems, but not previously reported for other Cr alloys.
\end{abstract}

DOI: 10.12693/APhysPolA.131.1132

PACS/topics: 75.50.Ee, 75.30.Fv, 74.70.Ad, 74.40.Kb

\section{Introduction}

Previous studies on the magnetic phase diagram (MPD) of $\mathrm{Cr}_{100-z} \mathrm{Os}_{z}$ focused on diluent concentrations up to $z \leq 2$ [1]. This is surprising as the MPDs of many $\mathrm{Cr}$ alloys with group- 8 metals, such as Re and Ru, show interesting properties, including quantum critical behavior (QCB) [2,3], as well as superconducting (SC) behavior possibly co-existing with antiferromagnetic (AFM) order [1]. In order to address this limitation in the literature with regards to the phase diagram of $\mathrm{Cr}$ with group8 metals a more recent study [4] focused on completing the magnetic phase diagram of $\mathrm{Cr}_{100-z} \mathrm{Os}_{z}$. As $z$ is increased, the commensurate spin-density-wave (CSDW) phase forms at the triple point concentration $z=z_{L} . T_{N}$ increases rapidly for $z>z_{L}$ and reaches a maximum at $z \approx 4$. The AFM order is completely suppressed for alloys with $z>13$ and these samples remain paramagnetic (PM) for all $T>2 \mathrm{~K}$.

QCB, as well as SC behavior in Cr alloy systems are of current interest [5-9]. SC properties in $\mathrm{Cr}-\mathrm{Os}$ alloys with very high concentrations of Os have previously been reported [10]. Alloys containing 28 and 33 at.\% Os show superconducting transition temperatures $\left(T_{C}\right)$ at 4.03 and $1.03 \mathrm{~K}$, respectively, while for the $\mathrm{Cr}_{75} \mathrm{Os}_{25}$ samples two $T_{C}$ values were obtained at 2.4 and $2.75 \mathrm{~K} \mathrm{[10].} \mathrm{This}$ is in agreement with results obtained for other $\mathrm{Cr}$ alloys with group-8 diluents that also show $\mathrm{SC}$, such as in the case of $\mathrm{Cr}-\mathrm{Ir}, \mathrm{Cr}-\mathrm{Ru}$ and $\mathrm{Cr}-\mathrm{Rh}[1,5,10]$. However, $\mathrm{Cr}$ alloys with high diluent concentrations of group- 8 metal does not exclusively show a body centered cubic (bcc) structure, but also include binary phases such as $\mathrm{Cr}_{3} \mathrm{X}$ with a cubic $A 15$ phase, shown to be SC [11-14].

*corresponding author; e-mail: alettap@uj.ac.za
The present study probes the behavior of the CSDWPM phase line on the $\mathrm{Cr}_{100-z} \mathrm{Os}_{z}$ MPD further, through resistivity $(\rho)$ and magnetization $(M)$ measurements, in order to investigate possible QCB and SC behavior in the region where this phase line is terminated.

\section{Experimental methods}

Polycrystalline binary $\mathrm{Cr}_{100-z} \mathrm{Os}_{z}$ alloys with $2 \leq z \leq$ 30.6 were prepared by arc melting in a purified low pressure argon atmosphere from $\mathrm{Cr}$ and Os of mass fractional purities $99.999 \%$ and $99.99 \%$, respectively. The actual elemental composition and homogeneity were confirmed using electron microprobe analyses. The experimental error in the actual concentrations is approximately $1 \%$. Powder X-ray diffraction (XRD) analyses, using $\mathrm{Cu} K_{\alpha}$ was used to characterize the samples structurally and confirm whether the samples were single or multiphase. Electrical resistivity and magnetization measurements were performed using a Cryogenic Ltd. ${ }^{3} \mathrm{He}$ measurement platform.

\section{Results}

In Fig. 1 the XRD patterns for the $\mathrm{Cr}_{86} \mathrm{Os}_{14}$ and $\mathrm{Cr}_{72} \mathrm{Os}_{28}$ alloys are shown. Comparing the relative peak positions obtained from the Joint Council of Powder Diffraction Database (JCPDD) for pure Cr bcc data base, to the current XRD spectra of $\mathrm{Cr}_{86} \mathrm{Os}_{14}$, it is evident that only the primary reflections (110), (200), (211) and (220) are seen. This was the case for all the samples with $z<22$. The absence of any other reflections indicates that these alloys are single phase. However, analysis of the XRD pattern of the $\mathrm{Cr}_{72} \mathrm{Os}_{28}$ sample indicate two predominant phases, the cubic $\mathrm{A} 15 \mathrm{Cr}_{3} \mathrm{Os}$ and the tetragonal $\mathrm{Cr}_{2}$ Os phases. Similar results were also found for the samples with $z>22$ and are in line with what is expected from the equilibrium chemical phase diagram of Cr-Os alloys as was previously reported by Venkataraman and Neumann [11]. 


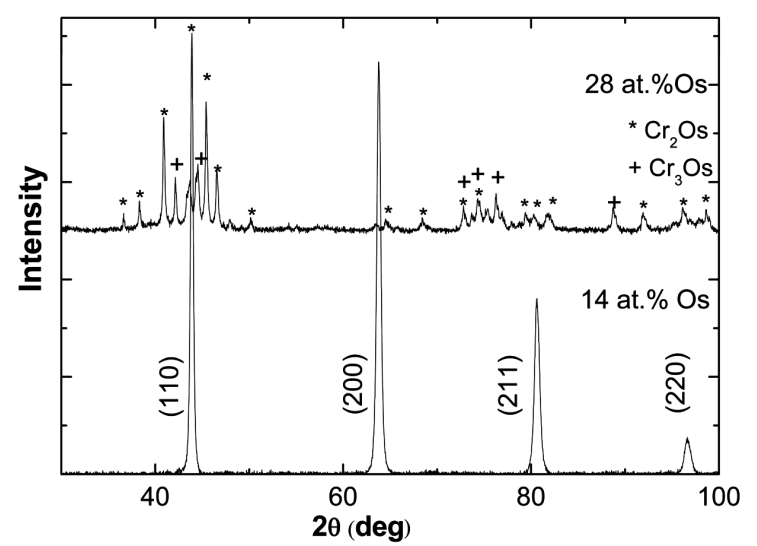

Fig. 1. XRD patterns for the $\mathrm{Cr}_{86} \mathrm{Os}_{14}$ and $\mathrm{Cr}_{72} \mathrm{O}_{28}$ alloys. The $(h k l)$ Miller indices of the reflections obtained from the JCPDD expected for the profile of Cr, $\mathrm{Cr}_{2} \mathrm{Os}$ and $\mathrm{Cr}_{3} \mathrm{Os}$ are indicated (Ref. no. 04-008-5187, 04-009-0319, and 04-009-0323).
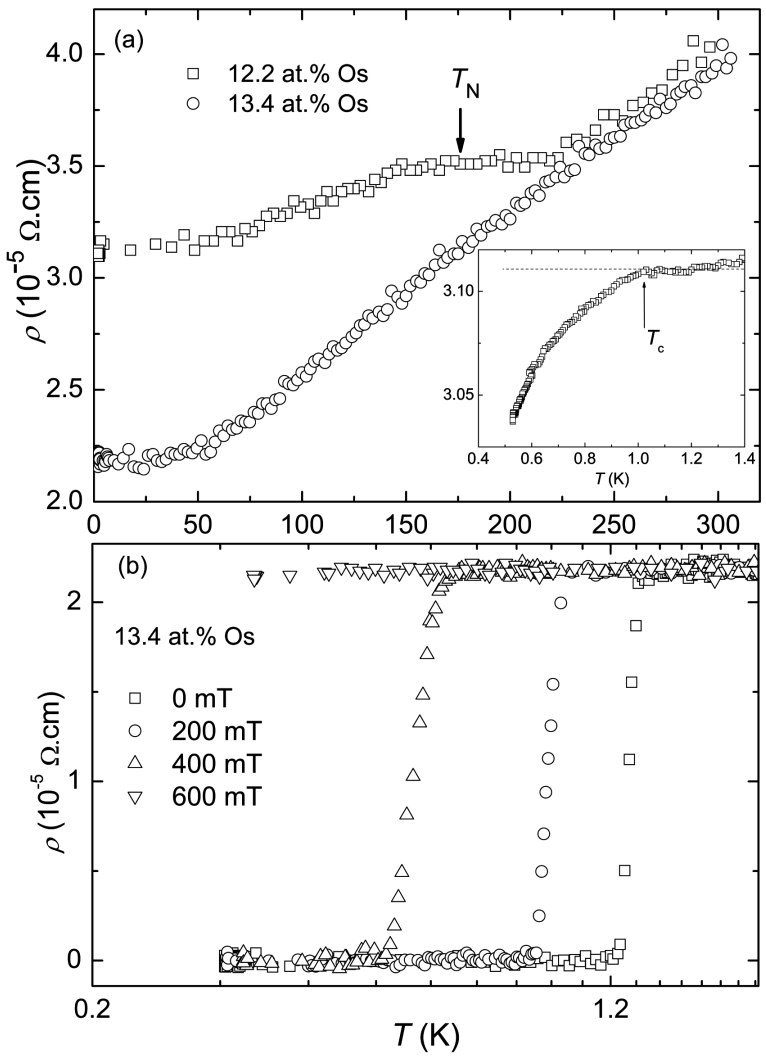

Fig. 2. (a) The electrical resistivity, $\rho$, of $\mathrm{Cr}_{100-z} \mathrm{Os}_{z}$ with $z=12.2(\square)$ and 13.4 (o) in the temperature range $2 \mathrm{~K} \leq T \leq 300 \mathrm{~K}$. The inset shows the $\rho(T)$ low temperature behavior for the $z=12.2$ sample. (b) Low temperature $\rho(T)$ behavior for the $z=13.4$ sample in various applied magnetic fields.

The electrical resistivity, $\rho$, of samples $z=12.2$ and 13.4 , in the temperature range $2 \mathrm{~K} \leq T \leq 300 \mathrm{~K}$, are shown in Fig. 2a. $\rho(T)$ curves for alloys with $2 \leq z \leq 12.2$ show well defined anomalies associated with the formation of the SDW on entering the AFM phase on cooling through the Néel temperature $\left(T_{N}\right)[1,4]$. Although the

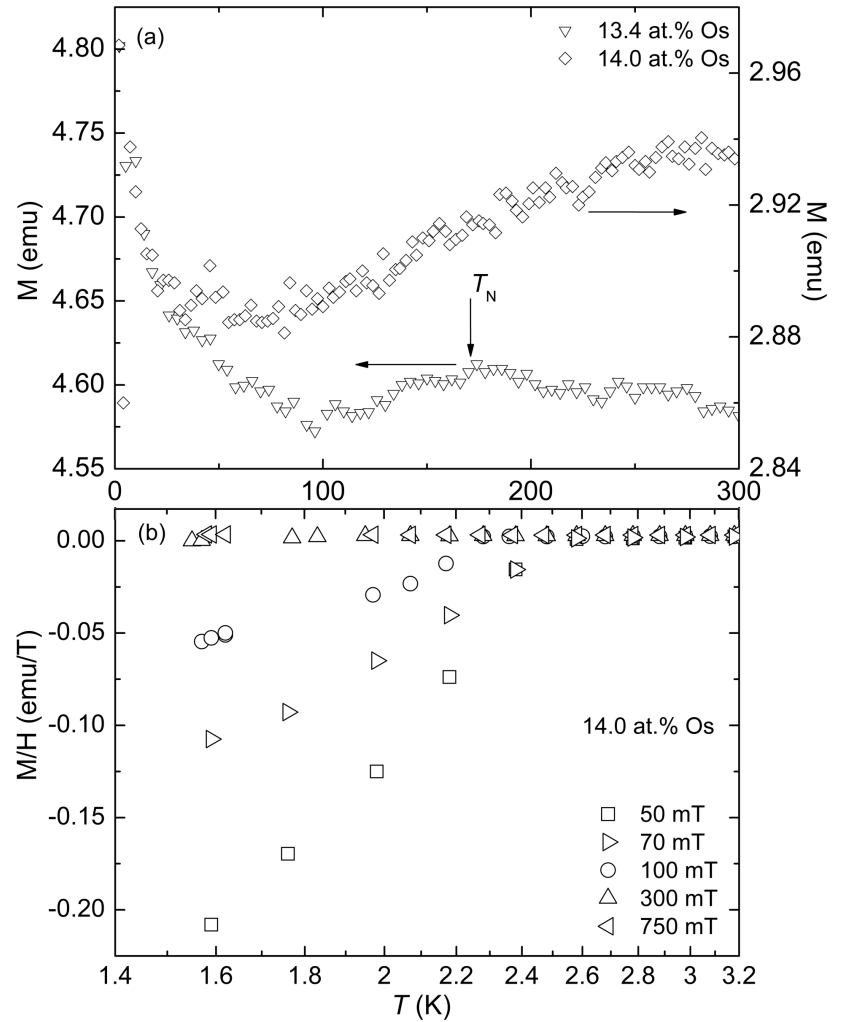

Fig. 3. (a) The magnetization, $M$, as function of temperature, $T$, for the $\mathrm{Cr}_{100-z} \mathrm{Os}_{z}$ samples with $z=$ $13.4(\nabla)$ and $14.0(\diamond)$ and $(\mathrm{b}) M / H$ versus $T$ for the $z=14.0$ sample.

addition of Os suppresses the size of this anomaly, a clear anomaly is still seen for the $z=12.2$ sample. However, this is not the case for the $z=13.4$ sample. The inset shows the low temperature behavior for $z=12.2$, indicating a downturn just above the low temperature limit of the apparatus. The SC temperature was taken for this alloy at the point where the resistance starts to decrease from its normal metallic behavior on cooling, indicating the onset of $\mathrm{SC}$ behavior at $T_{C}=1.0 \mathrm{~K}$. Figure $2 \mathrm{~b}$ shows the low temperature $\rho(T)$ behavior of the $z=13.4$ sample for various applied fields. In zero applied field a clear SC transition is seen for the $z=13.4$ sample in the form of a sharp decrease in $\rho(T)$ at $1.25 \mathrm{~K}$. An external magnetic field $\left(B=\mu_{0} H\right)$ is applied perpendicular to the direction of the current passed through the sample. With the application of magnetic field the onset of SC occurred at lower temperatures, as expected due to the pair breaking nature of the field on the Cooper pairs within the SC ground state. As the field strength is increased, a slight broadening of the transition width is seen, probably ascribed to vortex states appearing within a type-II superconductor. The SC behavior appears to be fully suppressed at $600 \mathrm{mT}$ and the sample remains metallic. The experimental error in the absolute value of $\rho$ is approximately $3 \%$ and originates mainly from errors in the determination of the sample dimensions. 
Figure 3a shows the magnetization $(M)$ versus temperature curves for the $z=13.4$ and 14.0 samples in the temperature range $2 \mathrm{~K} \leq T \leq 300 \mathrm{~K}$. Although no anomaly that can be associated with an AFM to PM transition was observed in the $\rho(T)$ curve of the $z=13.4$ sample, an anomaly in the form of a downturn on cooling is seen in the $M(T)$ curve of this sample, associated with $T_{N}$. This anomaly is however relatively weak, but of similar behaviour, to that observed [4] for samples with $z<13.4$.

The downturn in $M(T)$ on cooling through $T_{N}$ is ascribed to a decrease in the density of states at the Fermi surface when the nested parts of the electron and hole Fermi sheets are annihilated [1]. However, no such anomaly is observed for the $z=14.0$ sample, in line with what was reported for samples with $z \geq 14.0$ [4]. These samples were taken to be PM down to the lowest measurable temperature of the system. Figure $3 \mathrm{~b}$ shows $M / H(T)$ for the $z=14.0$ sample obtained in various applied fields, in the temperature range $300 \mathrm{mK} \leq T \leq$ $3.2 \mathrm{~K}$. A clear downturn is seen on cooling at $T_{C}=2.4 \mathrm{~K}$, where the AFM behavior gives way to SC. This transition is suppressed with the increase in applied magnetic field. $T_{C}$ was taken at the temperature associated with the onset of diamagnetism. Similar behavior was also seen for all samples with $z \geq 14.0$. The experimental error in the absolute value of $M$ is approximately $5 \%$.

\section{Discussions}

The $T_{N}$ and $T_{C}$ values obtained from the $\rho(T)$ and $M(T)$ measurements were used to prepare the MPD of the $\mathrm{Cr}_{100-z} \mathrm{Os}_{z}$ alloy system, shown in Fig. 4. The CSDW phase line terminates in a SC dome showing the existence of a possible quantum critical point, for which

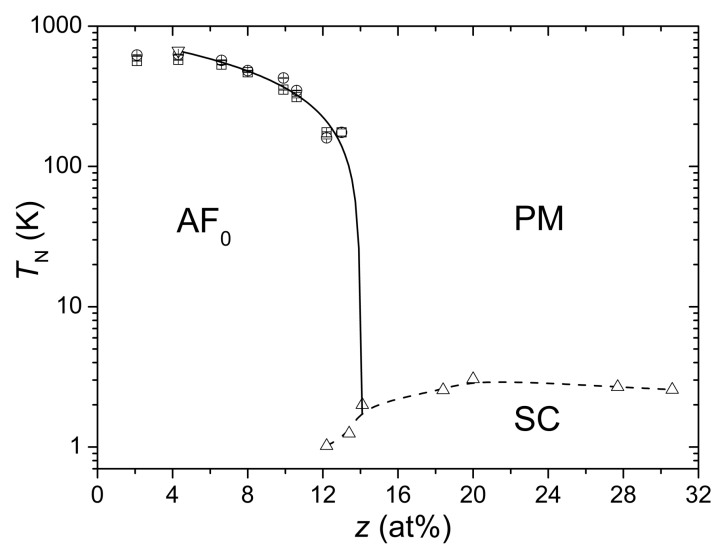

Fig. 4. The magnetic phase diagram for the $\mathrm{Cr}_{100-z} \mathrm{Os}_{z}$ alloy system as function of Os concentration, $z$, showing $T_{N}$ and $T_{C}$ values $(\square, \Delta)$ obtained from electrical resistivity and magnetization (o) measurements, with error bars. $\mathrm{PM}, \mathrm{AF}_{0}$ and $\mathrm{SC}$ refers to the paramagnetic, commensurate SDW and superconducting phases, respectively. The solid line is a power law fit to the data and dashed line a guide to the eye.
$T_{N}$ continuously tends to a zero at a critical concentration, $z_{c}$. The value of $z_{c}$ was obtained from a fit to the CSDW-PM phase line, using a power law of the form $T_{N}=a\left(z_{c}-z\right)^{b}$. The numerical values for these fitting parameters are: $a=130 \pm 60 \mathrm{~K}, b=0.69 \pm 0.19$ and $z_{c}=14.00 \pm 0.20$. The scaling component fits well to the mean-field value $b=0.5$, that is expected for doping induced quantum phase transitions $[2,3]$.

\section{Conclusions}

Present $\rho(T)$ results indicate that in the $z=12.2$ sample, that exhibits a bcc structure, both an PM-AFM and AFM-SC transition occur, indicating the co-existence of SDW antiferromagnetism and SC. The MPD obtained show that the $\mathrm{Cr}_{100-z} \mathrm{Os}_{z}$ system exhibit possible QCB and that the CSDW-PM phase line terminates in a SC dome, similar to that previously observed in certain heavy fermion systems [15]. Although it was previously suggested for the $\left(\mathrm{Cr}_{84} \mathrm{Re}_{16}\right)_{100-y} \mathrm{~V}_{y}$ system [5], this behaviour has now been confirmed for $\mathrm{Cr}_{100-z} \mathrm{Os}_{z}$ alloy system.

\section{Acknowledgments}

Funding from SA NRF (projects: 80928, 93551 and 88080 ), UJ URC/FRC, and use of UJ Spectrum is acknowledged.

\section{References}

[1] E. Fawcett, H.L. Alberts, V.Y. Galkin, D.R. Noakes, J.V. Yakhmi, Rev. Mod. Phys. 66, 25 (1994).

[2] L. Reddy, H.L. Alberts, A.M. Strydom, A.R.E. Prinsloo, A.M. Venter, J. Appl. Phys. 103, 07C903 (2008).

[3] B.S. Jacobs, A.R.E. Prinsloo, C.J. Sheppard, A.M. Strydom, J. Appl. Phys. 113, 17E126 (2013).

[4] P.R. Fernando, A.R.E. Prinsloo, C.J. Sheppard, L. Lodya, in: Proc. 58th Conf. of the South African Institute of Physics, Eds. R. Botha, T. Jili, I. Basson, Zululand 2013, p. 25.

[5] B.S. Jacobs, C.J. Sheppard, A.R.E. Prinsloo, L. Lodya, J. Appl. Phys. 115, 17E121 (2014).

[6] A. Yeh, Y-A. Soh, J. Brooke, G. Aeppli, T.F. Rosenbaum, S.M. Hayden, Nature 419, 459 (2002).

[7] M. Lee, A. Husmann, T.F. Rosenbaum, G. Aeppli, Phys. Rev. Lett. 92, 187201 (2004).

[8] R. Jaramillo, Y. Feng, J. Wang, T.F. Rosenbaum, Proc. Natl. Acad. Sci. USA 107, 09E116 (2010).

[9] C.J. Sheppard, A.R.E. Prinsloo, H.L. Alberts, A.M. Strydom, J. Appl. Phys. 109, 07E104 (2011).

[10] R.D. Blaugher, R.E. Hein, J.E. Cox, R.M. Waterstrat, J. Low. Temp. Phys. 1, 539 (1969).

[11] M. Venkataraman, J.P. Neumann, Bull. Alloy Phase Diagr. 11, 8 (1990).

[12] E.C. van Reuth, Acta Crystallogr. B 24, 186 (1968).

[13] R. Flukiger, A. Paoli, J. Muller, Solid State Commun. 14, 443 (1974).

[14] B.T. Matthias, T.H. Geballe, V.B. Compton, E. Corenzwit, G.W. Hull, Phys. Rev. 128, 588 (1962).

[15] H. Löhneysen, A. Rosch, V. Matthias, P. Wölfle, Rev. Mod. Phys. 79, 1015 (2007). 